\title{
Algorithm using Neuron-Specific Enolase and Pro- Gastrin-Releasing Peptide to Increase the Diagnostic Accuracy for Small Cell Lung Cancer
}

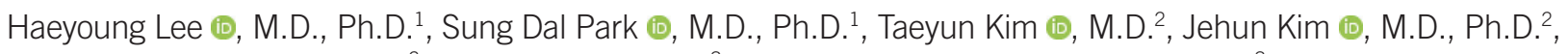

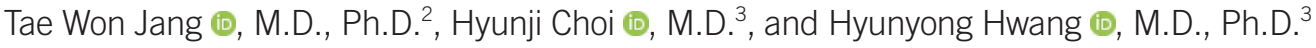 \\ ${ }^{1}$ Department of Thoracic and Cardiovascular Surgery, ${ }^{2}$ Division of Pulmonology, Department of Internal Medicine, ${ }^{3}$ Department of Laboratory Medicine, Kosin \\ University Gospel Hospital, Busan, Korea
}

\section{Dear Editor,}

Small cell lung cancer (SCLC) is an aggressive neuroendocrine tumor that accounts for approximately $15 \%$ of all lung cancers [1]. Because cancer cells in SCLC grow rapidly, the cancer likely has invaded other organs by the time symptoms present, which frequently delays initial treatment [2]. Therefore, early detection of SCLC is essential for improving the prognosis.

Chest X-ray and computed tomography (CT) are the diagnostic methods of choice in lung cancer. Although low-dose CT is recommended for lung cancer screening in high-risk populations, issues concerning its efficacy, high false-positive rates, overdiagnosis, significant costs, and radiation risk have been raised [3]. Therefore, it is important to focus on harmless biomarkers, and neuron-specific enolase (NSE) has been acknowledged as the most reliable tumor marker for SCLC [4].

Pro-gastrin-releasing peptide (ProGRP) has been used for SCLC screening in Korea since the fully automated ARCHITECT ProGRP immunoassay (Abbott Laboratories, Abbott Park, USA) was introduced in 2012. While ProGRP is a better SCLC marker than NSE [5], it is not as commonly used in clinical practice as NSE. This is likely because of the low serum stability of ProGRP [6]. In the Elecsys ProGRP assay (Roche Diagnostics Interna- tional, Rotkreuz, Switzerland) that was developed later, the ProGRP-stability issue was solved, and the assay showed clear benefits over the ARCHITECT ProGRP assay [7].

Although numerous studies have compared the validity of various tumor markers in SCLC diagnosis [8-10], no study has presented a practically available tool that uses NSE and ProGRP to increase the diagnostic accuracy. The goal of our study was to create an algorithm to differentiate SCLC from non-small cell lung cancer (NSCLC) using NSE and ProGRP. We prospectively analyzed serum samples from 100 lung cancer patients (age = $67.8 \pm 1.0$ years, male/female ratio $=2.85$ ), including 24 SCLC and 76 NSCLC patients (adenocarcinoma [N=47], squamous cell carcinoma [N=25], large-cell carcinoma $[\mathrm{N}=3]$, and adenosquamous carcinoma [N=1]), for NSE and ProGRP. The Institutional Review Board of Kosin University Gospel Hospital, Busan, Korea, approved the study protocol (KUGH 2015-06106). Informed consent was obtained from the patients. NSE (Elecsys NSE, Roche Diagnostics International) and ProGRP (Elecsys ProGRP, Roche Diagnostics International) levels were analyzed using the Cobas e601 module (Roche Diagnostics International).

The area under the receiver operating characteristic (ROC)
Received: May 26, 2020

Revision received: July 20, 2020

Accepted: November 13, 2020

Corresponding author: Hyunyong Hwang, M.D., Ph.D.

Department of Laboratory Medicine, Kosin University Gospel Hospital,

262 Gamcheon-ro, Seo-gu, Busan 49267, Korea

Tel: +82-51-990-6371, Fax: +82-51-990-3034

E-mail: terminom@hanmail.net

\section{(c) $(1) \Theta$}

(C) Korean Society for Laboratory Medicine

This is an Open Access article distributed under the terms of the Creative Commons Attribution Non-Commercial License (https://creativecommons.org/licenses/by-nc/4.0) which permits unrestricted non-commercial use, distribution, and reproduction in any medium, provided the original work is properly cited. 
curve, sensitivity, specificity, and diagnostic accuracy results are presented in Table 1. ProGRP level showed excellent performance in differentiating between SCLC and NSCLC at a cutoff value of $85.70 \mathrm{pg} / \mathrm{mL}$ but had a relatively weak sensitivity, whereas NSE level showed excellent sensitivity but very low specificity at a cutoff value of $17.30 \mathrm{ng} / \mathrm{mL}$. This indicates that NSE level alone may lead to false-positive results at a cutoff value of $17.30 \mathrm{ng} / \mathrm{mL}$ and that it cannot accurately differentiate between SCLC and NSCLC.

We created an algorithm using NSE and ProGRP to increase the diagnostic accuracy for SCLC (Fig. 1). When ProGRP and NSE results differed, the final result was decided by the algorithm. If the NSE level was greater than $14.24 \mathrm{ng} / \mathrm{mL}$ (the cutoff value for $100 \%$ sensitivity based on ROC curve analysis) in the ProGRP (+)/NSE (-) group or $83.97 \mathrm{ng} / \mathrm{mL}$ (mean plus three times the SD of NSE level in the NSCLC group) in the ProGRP $(-) /$ NSE (+) group, a positive result was reported. By applying this algorithm, we could improve the relatively low sensitivity of ProGRP level from $75 \%$ to $87.5 \%$, with a specificity of $94.7 \%$. The accuracy improved to $93 \%$ from $90 \%$ for ProGRP level and $49 \%$ for NSE level.

The algorithm was created considering the limited number of lung cancer patients and is based on the NSE and ProGRP levels from our hospital. The cutoff values of NSE and ProGRP levels could vary in each institution. We did not evaluate the NSE and ProGRP levels in normal control or other cancer groups; these need to be further studied.
In conclusion, an elevated NSE level is observed in many NSCLC patients. NSE level alone is not sufficient to accurately differentiate between SCLC and NSCLC. To improve the diagnostic power and accuracy for SCLC, ProGRP level needs to be additionally tested. Sequential testing of NSE and ProGRP levels and application of a diagnostic algorithm could increase the ac-

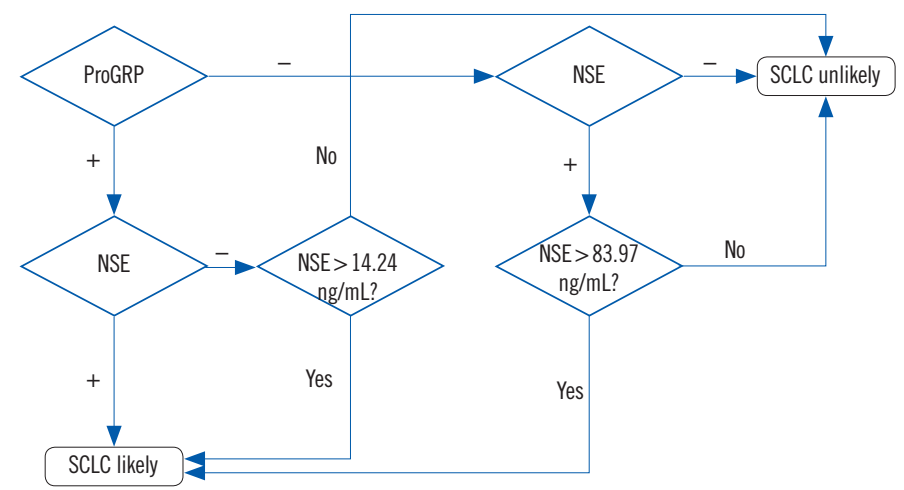

Fig. 1. Algorithm for differentiating between SCLC and NSCLC. The cutoff value for ProGRP is $85.70 \mathrm{pg} / \mathrm{mL}$, and that for NSE is 17.30 $\mathrm{ng} / \mathrm{mL}$. When a discordant result is observed between ProGRP and NSE, two cutoff values are used. One of the values is $14.24 \mathrm{ng} / \mathrm{mL}$, which is the cutoff value for $100 \%$ sensitivity of NSE based on the ROC curve analysis, and the other is $83.97 \mathrm{ng} / \mathrm{mL}$, which is the mean value plus 3SD of NSE in the NSCLC patient group. We calculated these values based on the test results of 100 patients. Therefore, these values could be adjusted based on the evaluated patient groups in each institution.

Abbreviations: NSE, neuron-specific enolase; ProGRP, pro-gastrin- releasing peptide; SCLC, small cell lung cancer.

Table 1. Sensitivity, specificity, accuracy, and AUC of NSE, ProGRP, and the proposed algorithm for SCLC

\begin{tabular}{|c|c|c|c|c|c|c|c|c|c|c|c|}
\hline \multirow{2}{*}{ Test } & & \multicolumn{3}{|c|}{ SCLC (N) } & \multirow{2}{*}{$\operatorname{Sn}(\%)$} & \multirow{2}{*}{ Sp (\%) } & \multirow{2}{*}{ Ac (\%) } & \multirow{2}{*}{ Cut-off } & \multirow{2}{*}{ Mean (SD) } & \multirow{2}{*}{ AUC (95\% Cl) } & \multirow{2}{*}{$P$} \\
\hline & & + & - & Total & & & & & & & \\
\hline \multirow[t]{3}{*}{ NSE } & + & 23 & 50 & 73 & 95.8 & 34.2 & 49.0 & $17.30 \mathrm{ng} / \mathrm{mL}$ & $38.27(37.84) \mathrm{ng} / \mathrm{mL}$ & $0.847(0.760-0.935)$ & $<0.001$ \\
\hline & - & 1 & 26 & 27 & & & & & & & \\
\hline & Total & 24 & 76 & 100 & & & & & & & \\
\hline \multirow[t]{3}{*}{ ProGRP } & + & 18 & 4 & 22 & 75.0 & 94.7 & 90.0 & $85.70 \mathrm{pg} / \mathrm{mL}$ & $374.59(1,279.93) \mathrm{pg} / \mathrm{mL}$ & $0.867(0.766-0.969)$ & $<0.001$ \\
\hline & - & 6 & 72 & 78 & & & & & & & \\
\hline & Total & 24 & 76 & 100 & & & & & & & \\
\hline \multirow[t]{3}{*}{ Algorithm } & + & 21 & 4 & 25 & 87.5 & 94.7 & 93.0 & $14.24 \mathrm{ng} / \mathrm{mL}$ & - & - & - \\
\hline & - & 3 & 72 & 75 & & & & $83.97 \mathrm{ng} / \mathrm{mL}$ & & & \\
\hline & Total & 24 & 76 & 100 & & & & & & & \\
\hline
\end{tabular}

The percentage of positive results for NSE was $68.06 \%$ (49/72) in NSCLC and 31.94\% (23/72) in SCLC. The mean \pm SD of NSE level in SCLC was significantly greater than that in NSCLC $(76.1 \pm 54.7$ vs. $26.3 \pm 19.2 ; P<0.001)$; this explains the low specificity for NSE. When the 2 -by-2 table for estimating the diagnostic accuracy of NSE, ProGRP, and algorithm test results was prepared, the positive test results were categorized into true positives and false positives, and negative results into true negatives and false negatives. Diagnostic accuracy was defined as the fraction of true positives and true negatives derived from all classifications.

Abbreviations: SCLC, small cell lung cancer; NSCLC, non-small cell lung cancer; NSE, neuron-specific enolase; ProGRP, pro-gastrin-releasing peptide; Sn, sensitivity; Sp, specificity; Ac, diagnostic accuracy; SD, standard deviation; AUC, the area under the receiver operating characteristic curve; Cl, confidence interval. 
curacy of SCLC diagnosis. Further studies assessing the clinical usefulness of this algorithm by comparing routine diagnostic processes using radiological and histological methods with the present algorithm are warranted.

\section{AUTHOR CONTRIBUTIONS}

Conceptualization: Hwang $\mathrm{H}$ and Lee $\mathrm{H}$; data curation: Choi $\mathrm{H}$ and Lee H; methodology: Jang TW, Park SD, and Kim T; validation: Hwang $\mathrm{H}$; writing-original draft: Lee $\mathrm{H}$; writing-review \& editing: Hwang $\mathrm{H}$.

\section{CONFLICTS OF INTEREST}

None declared.

\section{RESEARCH FUNDING}

This work was supported by the Research Program funded by Roche Diagnostics.

\section{ORCID}

Haeyoung Lee Sung Dal Park Jehun Kim Taeyun Kim Tae Won Jang Hyunji Choi https://orcid.org/0000-0003-4972-3608 https://orcid.org/0000-0002-3344-9803 https://orcid.org/0000-0002-2594-0256 https://orcid.org/0000-0001-7786-5051 https://orcid.org/0000-0001-8075-8097 https://orcid.org/0000-0002-6453-7099
Hyunyong Hwang

https://orcid.org/0000-0003-0662-3041

\section{REFERENCES}

1. Gazdar AF, Bunn PA, Minna JD. Small-cell lung cancer: what we know, what we need to know and the path forward. Nat Rev Cancer 2017;17: 725-37.

2. Yang S, Zhang Z, Wang Q. Emerging therapies for small cell lung cancer. J Hematol Oncol 2019;12:47.

3. Shen H. Low-dose CT for lung cancer screening: opportunities and challenges. Front Med 2018;12:116-21.

4. Isgrò MA, Bottoni P, Scatena R. Neuron-specific enolase as a biomarker: biochemical and clinical aspects. Adv Exp Med Biol 2015;867:12543.

5. Nisman B, Biran H, Ramu N, Heching N, Barak V, Peretz T. The diagnostic and prognostic value of ProGRP in lung cancer. Anticancer Res 2009;29:4827-32.

6. Kim HR, Oh IJ, Shin MG, Park JS, Choi HJ, Ban HJ, et al. Plasma pro GRP concentration is sensitive and specific for discriminating small cell lung cancer from nonmalignant conditions or non-small cell lung cancer. J Korean Med Sci 2011;26:625-30.

7. Korse CM, Holdenrieder S, Zhi XY, Zhang X, Qiu L, Geistanger A, et al. Multicenter evaluation of a new progastrin-releasing peptide (ProGRP) immunoassay across Europe and China. Clin Chim Acta 2015;438:38895.

8. Liu L, Teng J, Zhang L, Cong P, Yao Y, Sun G, et al. The combination of the tumor markers suggests the histological diagnosis of lung cancer. Biomed Res Int 2017;2017:2013989.

9. Oya Y, Yoshida T, Uemura T, Murakami Y, Inaba Y, Hida T. Serum Pro GRP and NSE levels predicting small cell lung cancer transformation in a patient with ALK rearrangement-positive non-small cell lung cancer: A case report. Oncol Lett 2018;16:4219-22.

10. Yang DW, Zhang Y, Hong QY, Hu J, Li C, Pan BS, et al. Role of a serumbased biomarker panel in the early diagnosis of lung cancer for a cohort of high-risk patients. Cancer 2015;121(S17):3113-21. 